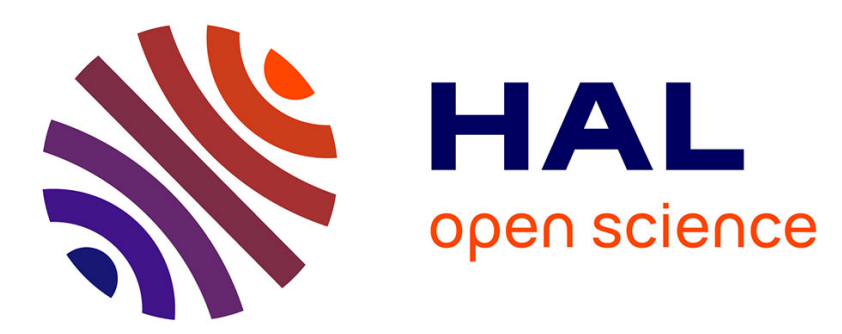

\title{
Cathodoluminescence and photoluminescence comparative study of erbium-doped silicon-rich silicon oxide
}

Sébastien Cueff, Christophe Labbé, Benjamin Dierre, Julien Cardin, Larysa

Khomenkova, Fabbri Filippo, Takashi Sekiguchi, Richard Rizk

\section{To cite this version:}

Sébastien Cueff, Christophe Labbé, Benjamin Dierre, Julien Cardin, Larysa Khomenkova, et al.. Cathodoluminescence and photoluminescence comparative study of erbium-doped silicon-rich silicon oxide. Journal of Nanophotonics, 2011, 5 (1), pp.051504. 10.1117/1.3549701 . hal-01139282

\section{HAL Id: hal-01139282 \\ https://hal.science/hal-01139282}

Submitted on 25 Apr 2017

HAL is a multi-disciplinary open access archive for the deposit and dissemination of scientific research documents, whether they are published or not. The documents may come from teaching and research institutions in France or abroad, or from public or private research centers.
L'archive ouverte pluridisciplinaire HAL, est destinée au dépôt et à la diffusion de documents scientifiques de niveau recherche, publiés ou non, émanant des établissements d'enseignement et de recherche français ou étrangers, des laboratoires publics ou privés. 


\title{
Cathodoluminescence and photoluminescence comparative study of erbium-doped silicon-rich silicon oxide
}

\author{
Sébastien Cueff, ${ }^{\text {a }}$ Christophe Labbé, ${ }^{a}$ Benjamin Dierre, ${ }^{b}$ Julien Cardin, ${ }^{a}$ \\ Larysa Khomenkova, ${ }^{a}$ Filippo Fabbri, ${ }^{b}$ Takashi Sekiguchi, ${ }^{b}$ \\ and Richard Rizk ${ }^{a}$ \\ ${ }^{a}$ Université de Caen, Centre de Recherche sur les Ions, les Matériaux et la Photonique \\ (CIMAP), ENSICAEN, CNRS, CEA/IRAMIS, 14050 CAEN cedex, France \\ ${ }^{\mathrm{b}}$ National Institute for Materials Science, 1-1 Namiki, Tsukuba, Ibaraki 305-0044, Japan \\ seb.cueff@gmail.com
}

\begin{abstract}
We present a study on erbium (Er)-doped silicon (Si)-rich silicon oxide thin films grown by the magnetron cosputtering of three confocal cathodes according to the deposition temperature and the annealing treatment. It is shown that, through a careful tuning of both deposition and annealing temperatures, it is possible to engineer the fraction of agglomerated Si that may play the role of sensitizer toward Er ions. To investigate the different emitting centers present within the films according to the fraction of agglomerated $\mathrm{Si}$, a cathodoluminescence experiment was made. We observe in all samples contributions from point-defect centers due to some oxygen vacancies and generally known as silicon-oxygen deficient centers (SiODC), at around 450-500 nm. The behavior of such contributions suggests the possible occurrence of an energy transfer from the SiODCs toward $\mathrm{Er}^{3+}$ ions. Photoluminescence experiments were carried out to characterize the energy transfer from Si nanoclusters toward $\mathrm{Er}^{3+}$ ions with a nonresonant wavelength $(476 \mathrm{~nm})$ that is unable to excite SiODCs and then exclude any role of these centers in the energy transfer process for the PL experiments. Accordingly, it is shown that structural differences have some effects on the optical properties that lead to better performance for hightemperature deposited material. This aspect is illustrated by the Er-PL efficiency that is found higher for $500^{\circ} \mathrm{C}$-deposited, when compared to that for RT-deposited sample. Finally, it is shown that the Er-PL efficiency is gradually increasing as a function of the fraction of agglomerated silicon.
\end{abstract}

Keywords: erbium; silicon-nanoclusters; thin films; energy transfer; photoluminescence; cathodoluminescence.

\section{Introduction}

The integrated circuit was invented fifty years ago and has continuously improved ever since. Microelectronics industries can now routinely integrate one billion transistors on a single chip. This tremendous progress is now facing several major issues lying mainly in increased signal delay and higher power dissipation, referred to as "interconnection bottleneck." Such problems associated with the electrical and thermal parasites could be overcome if the present metallic interconnections are replaced by photonics devices. Recently, a fully silicon-based photonics device was demonstrated for interchip communications. ${ }^{1}$ Nevertheless, for intrachips communications, interconnections must be integrated into microprocessors and a smart choice would be to use the "less-absorbed wavelength in silica," such as the standard $1.5 \mu \mathrm{m}$ to transport the 
information. For this purpose, erbium (Er) ion in silica is a good candidate as an emitting center at the standard $1.5 \mu \mathrm{m}$, corresponding to the ${ }^{4} I_{13 / 2} \rightarrow{ }^{4} I_{15 / 2}$ transitions within a $4 \mathrm{f}$ shell. Unfortunately, this rare earth is very weakly excited in silica, with an absorption cross section $\sigma$ as low as $10^{-20}-10^{-21} \mathrm{~cm}^{2}$ that prevents the achievement of optical gain within compact devices. However, it was shown and demonstrated that this low absorption can be effectively increased by a factor as high as $10^{4}$, when $\mathrm{Er}^{3+}$ ions are excited through the neighboring Si-nanoclusters (Si-nc) that absorb the excitation photons along a broad band with a cross section close to $10^{-16} \mathrm{~cm}^{2} .^{2-5}$ Such findings led the scientific community to consider the erbium-doped silicon-rich silicon oxide (SRSO:Er) as a promising material for integrated photonics, although few studies reported some internal optical gain. ${ }^{6,7}$ Two main factors are considered as limiting the achievement of a net gain: 1. the low fraction of Er coupled to Si-nc that stems from the distance-dependent energy transfer between $\mathrm{Er}^{3+}$ ions and Si-nc sensitizers with a critical distance lower than $1 \mathrm{~nm},{ }^{8-10}$ and 2. the low fraction of optically active $\mathrm{Er}^{3+}$ ions. ${ }^{11,12}$ Recently, our group demonstrated a way to increase the fraction of coupled Er, from $2-3 \%$ to about $22 \%$, by carefully optimizing the concentrations of Si-excess and erbium ${ }^{13}$ and also showed the importance of the temperature of deposition for increasing the number of optically active $\mathrm{Er}^{3+}$ ions. ${ }^{14}$ Such advances are still, however, insufficient to achieve a net optical gain, which require the coupling of the majority of Er ions to the sensitizers. The latter are not well understood, especially as the Si-entities able to sensitize $\mathrm{Er}^{3+}$ ions appear in different states, from atomic-scale centers ${ }^{15,16}$ to well-defined Si-nanocrystals ${ }^{17,18}$ as well as amorphous Si-nanoclusters. ${ }^{5,13,19}$ The purpose of this paper is to investigate the nature of the Si-based sensitizers considered as responsible of an energy transfer toward $\mathrm{Er}^{3+}$ ions. By successively tuning the deposition and annealing temperatures, we were able to obtain a wide range of different state of Si-based sensitizers. A combination of emission dispersive $\mathrm{x}$ ray spectroscopy (EDX) and Fourier-transform infrared (FTIR) spectroscopy allows us to characterize the Si-based entities in term of percentage of agglomerated Si. Thanks to cathodoluminescence (CL) spectroscopy, the different emitting centers present in the films were identified, such as silicon-oxygen deficient centers (SiODCs) that were observed in all of our samples. To avoid any influence from SiODCs on the energy transfer, photoluminescence

(PL) experiments were carried out using a $476 \mathrm{~nm}$ excitation wavelength that is nonresonant for $\mathrm{Er}^{3+}$ ions and also below the excitation threshold for SiODCs.

\section{Experimental Details}

The samples used for this study were deposited by the magnetron cosputtering of three confocal cathodes: $\mathrm{SiO}_{2}, \mathrm{Si}$, and $\mathrm{Er}_{2} \mathrm{O}_{3}$. The power density applied on each of these cathodes can be independently tuned and are fixed at: $\mathrm{P}_{\mathrm{SiO} 2} \approx 8.88 \mathrm{~W} / \mathrm{cm}^{2}, \mathrm{P}_{\mathrm{Si}} \approx 1.63 \mathrm{~W} / \mathrm{cm}^{2}$, and $\mathrm{P}_{\mathrm{Er} 203}$ $\approx 0.44 \mathrm{~W} / \mathrm{cm}^{2}$. The total plasma pressure was kept constant at $2 \mathrm{mTorr}$ during all deposition runs. The sample holder can be intentionally heated during the deposition and thus the deposition temperature, $T_{\mathrm{d}}$, was varied from room temperature (RT, i.e., $\sim 20^{\circ} \mathrm{C}$ ) to $700^{\circ} \mathrm{C}$. The substrate holder is rotated during the growth process, in order to ensure a good homogeneity for both composition and thickness. The duration for all depositions was kept constant at $10 \mathrm{~h}$. Some samples were submitted to a postdeposition annealing during $1 \mathrm{~h}$ under a flux of pure nitrogen. The annealing temperature, $T_{\mathrm{a}}$, was varied from 600 to $1100^{\circ} \mathrm{C}$.

The thickness was determined by the M-lines technique. The Er content and the stoichiometry of $\mathrm{SiO}_{x}$ matrix were measured by EDX. The FTIR absorption spectra were recorded under normal incidence to investigate the evolution of the $\mathrm{TO}_{3}$ peak, which provides some insights on the structural properties, such as the degree of phase separation and a rough estimate of $\mathrm{Si}$ excess.

CL spectra were obtained using a scanning electron microscope with CL system under high vacuum system. The electron beam is provided by a thermal field emission scanning electron microscope (Hitachi S4200) equipped with a CL system ${ }^{20}$ whose beam diameter was around tens of $\mathrm{nm}$. The electron energy and beam current were kept constant at $10 \mathrm{kV}$ and $4 \mathrm{nA}$, respectively, hence allowing appropriate comparison between the different samples. The light emitted by the sample is focused to an optical fiber and guided through the monochromator 
(Jobin-Yvon Triax 320). The signal is thus detected by a charge coupled device of 2048 channels (CCD, Jobin-Yvon Spectrum One). The excitation time was kept the same (10 s) for all samples and the recorded spectra were automatically converted into counts per seconds (cps).

PL measurements were carried out using the nonresonant $476 \mathrm{~nm}$ wavelength of an $\mathrm{Ar}^{+}$ laser, in order to ensure that the $\mathrm{Er}^{3+}$ ions are indirectly excited. The spot of the laser beam was carefully determined by a "moving knife-edge" method and was found to be around $3 \mathrm{~mm}^{2}$ at $1 / \mathrm{e}^{2}$ of the maximum intensity. For the visible PL assumed to originate from the Si nanoclusters, the emitted light is detected by photomultiplier tube (Hamamatsu). For the Er-related PL in the IR range $(1.53 \mu \mathrm{m})$, the emission is guided through a $1 \mathrm{~m}$ single-grating monochromator (Jobin Yvon) and collected by a liquid-nitrogen-cooled Germanium detector (Northcoast). All PL measurements were made using the standard lock-in technique with a chopper setting the reference frequency at $10 \mathrm{~Hz}$.

\section{Results and Discussion}

\subsection{Compositional Characteristics and Structural Properties}

The influence of the deposition temperature, $T_{\mathrm{d}}$, on the properties of 300-400 nm-thick films was already analyzed in our previous recent study.$^{14}$ However, the present deposited films are much thicker $(1.3-1.8 \mu \mathrm{m})$ what may result in several quantitative differences. Figure 1 shows the evolution of the thickness, the Er content and the stoichiometry parameter, i.e., the ratio $x=$ [O]/[Si], in function of $T_{\mathrm{d}}$.

It appears that the thickness is almost constant for $T_{\mathrm{d}} \leq 300^{\circ} \mathrm{C}$, before progressively decreasing for $T_{\mathrm{d}}>300^{\circ} \mathrm{C}$. In parallel, the Er content continuously decreases for $T_{\mathrm{d}}$ higher than $300^{\circ} \mathrm{C}$, and loses one order of magnitude when $T_{\mathrm{d}}$ reaches $700^{\circ} \mathrm{C}$. Concerning the composition of the as-deposited samples investigated by EDX, the stoichiometry parameter $x$ decreases by about $10 \%$ when $T_{\mathrm{d}}$ exceeds $300^{\circ} \mathrm{C}$, reaches a minimum of about 1.4 for $T_{\mathrm{d}}=500^{\circ} \mathrm{C}$, and then slightly increases.

Besides, $T_{\mathrm{d}}$ is likely to also influence the surface mobility of the deposited species during the deposition process, hence favoring the formation of Si agglomerates that may play the role of sensitizers toward the Er ions, when $T_{\mathrm{d}}$ is increased. Though this phenomenon was assumed

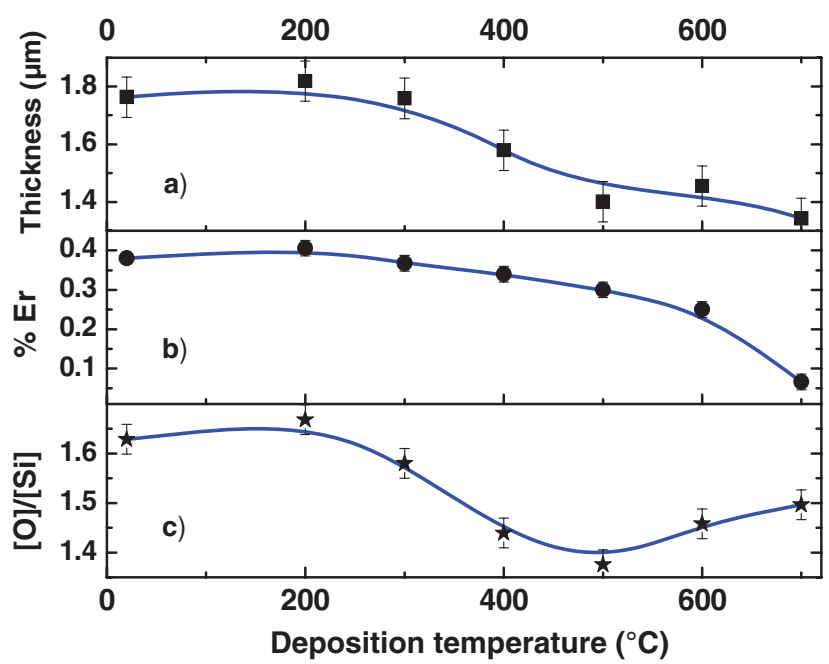

Fig. 1 Evolution of different compositional parameters as a function of the deposition temperature (a) thickness as measured by M-Lines, (b) erbium content, and (c) stoichiometric parameter $x=[\mathrm{O}] /[\mathrm{Si}]$. 
to occur in our previous study, ${ }^{14}$ it was not investigated. To fulfill this need, we made use of two complementary methods: FTIR method able to uniquely detect the Si bonded to O (see below) and the EDX that is capable of measuring the total silicon excess from the following relation:

$$
\mathrm{SiO}_{x} \rightarrow \frac{x}{2} \mathrm{SiO}_{2}+\left(1-\frac{x}{2}\right) \mathrm{Si},
$$

with $x$ being the stoichiometric parameter (i.e., the ratio [O]/[Si] as measured by EDX). We can, therefore, estimate the total $\mathrm{Si}$ excess in atomic percent by

$$
\% \mathrm{Si}_{\text {Excess }}=100 *[1-(x / 2)] /[1+x] .
$$

However, to get the fraction of agglomerated $\mathrm{Si}$, we used the FTIR spectroscopy method that is based on the evolution of the $\mathrm{TO}_{3}$ peak, ${ }^{21}$ which is related to the amount of $\mathrm{Si}$ unbonded to another $\mathrm{Si}$ atom, but rather bonded to oxygen. With this method, as soon as some Si-excess atoms are agglomerated, the "stoichiometric" parameter $y$ (i.e., the ratio $[\mathrm{O}] /[\mathrm{Si}]$ obtained by FTIR) is different from the parameter $x$ obtained by EDX. Consequently, the Si-excess content, as measured by FTIR, is underestimated when agglomeration of Si atoms occur (i.e., $y>x$ ). The parameter $y$ corresponds therefore to a $\mathrm{SiO}_{y}$ matrix composed of isolated atoms of $\mathrm{Si}$ excess bonded to oxygen within $\mathrm{SiO}_{2}$ matrix. This parameter $y$ of $\mathrm{SiO}_{y}$ is linked to the wavenumber value of the $\mathrm{TO}_{3}$ peak $\left(v_{T 03}^{S R S O: E r}\right)$ by the following linear relationship:

$$
v_{T 03}^{S R S O: E r}=A \cdot y+v_{T 03}^{S i} .
$$

For $\mathrm{SiO}_{2}, y=2$, we obtain

$$
A=\frac{v_{T 03}^{r e f S i O 2}-v_{T 03}^{S i}}{2} .
$$

The parameter $y$ can then be deduced from

$$
y=2 \cdot \frac{v_{T 03}^{S R S O: E r}-v_{T 03}^{S i}}{v_{T 03}^{r e f S i O 2}-v_{T 03}^{S i}} .
$$

Knowing the value for $v_{T 03}^{S i}\left(960 \mathrm{~cm}^{-1}\right.$ as measured by FTIR on Si substrate), and measuring $v_{T 03}^{r e f S i O 2}$ and $v_{T 03}^{S R O: E r}$ for each temperature of deposition (see Fig. 2), one can obtain the value

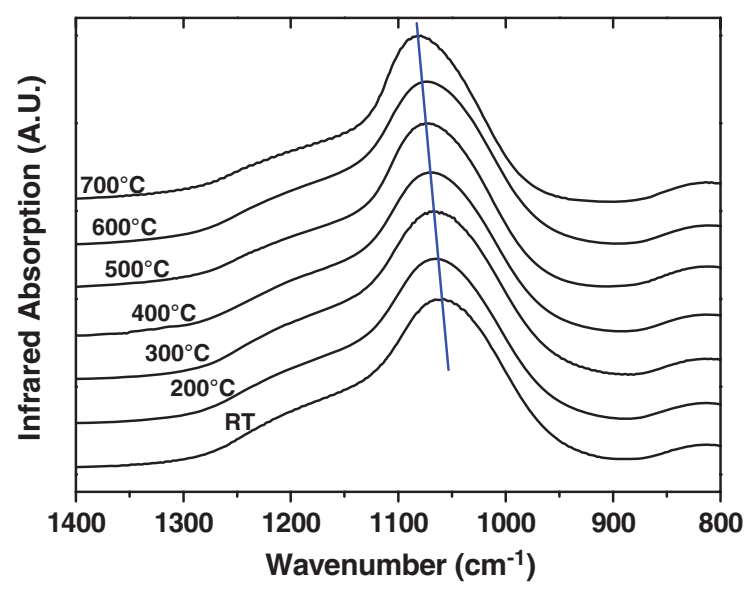

Fig. 2 FTIR absorption spectra of the as-deposited samples for all $T_{d}$. The blue line indicates the shift of the $\mathrm{TO}_{3}$ peak toward high wavenumber when $\mathrm{T}_{\mathrm{d}}$ is raised. Spectra were vertically shifted for clarity. 
of $y$ for each $T_{\mathrm{d}}$. With this value $y$ measured by FTIR, we obtain the atomic percentage of "isolated" silicon atom by the following formula:

$$
\% S i_{\text {isolated }}=100 \times \frac{\frac{1-y}{2}}{1+y} .
$$

Note that in Ref. 14, the estimated Si-excess represents a percentage of deviation from the stoichiometry of $\mathrm{SiO}_{2}$ and not an atomic percentage.

Any difference between values obtained by EDX and FTIR should thus be linked to an agglomeration phenomenon. Therefore, the comparison of the amounts of Si excess obtained by EDX and FTIR enables the determination of the fraction of agglomerated Si that can be estimated from the following relation:

$$
\mathrm{Si}_{x} \rightarrow \frac{x}{y} \mathrm{SiO}_{Y}+\frac{y-x}{y} \mathrm{Si} .
$$

The fraction of agglomerated $\mathrm{Si}$ is thus obtained by the following definition:

$$
\% S i_{\text {agglomerated }}=100 \times \frac{\frac{y-x}{y}}{1+x},
$$

which verify the equation

$$
\% \mathrm{Si}_{\text {Total Excess }}=\% \mathrm{Si}_{\text {agglomerated }}+\% \mathrm{Si}_{\text {isolated }} \text {. }
$$

The evolution of this fraction against $T_{d}$ [Eq. (8)] is compared in Fig. 3 to that of the total $\mathrm{Si}$ excess, as determined by EDX [Eq. (2)].

It is noteworthy here that we are dealing with the atomic percentage of $\mathrm{Si}$ excess whose atoms are linked to each other within Si agglomerates. Such a fraction is obviously different from the number of sensitizers or Si-nc that should be lower than the percentage of Si atoms bonded only to other $\mathrm{Si}$ atoms. As expected, the fraction of agglomerated Si-excess is always lower than the total excess-silicon, while both show a clear increase when $T_{\mathrm{d}}$ is raised beyond $300^{\circ} \mathrm{C}$ until reaching a maximum for $T_{\mathrm{d}}=500^{\circ} \mathrm{C}$. The values of both parameters join at about $10 \%$ for the highest $T_{\mathrm{d}}$ value $\left(700^{\circ} \mathrm{C}\right)$. At this stage, several observations can be made: 1 . increasing the deposition temperature leads to a progressive agglomeration of the Si-excess, 2. the difference

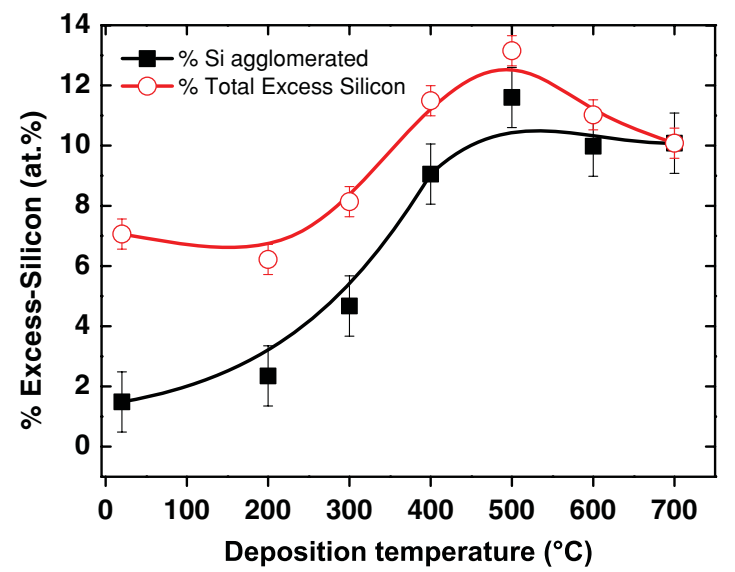

Fig. 3 Compared evolution of the total excess-silicon as measured by EDX (empty circles) and the agglomerated excess-silicon (full square). 


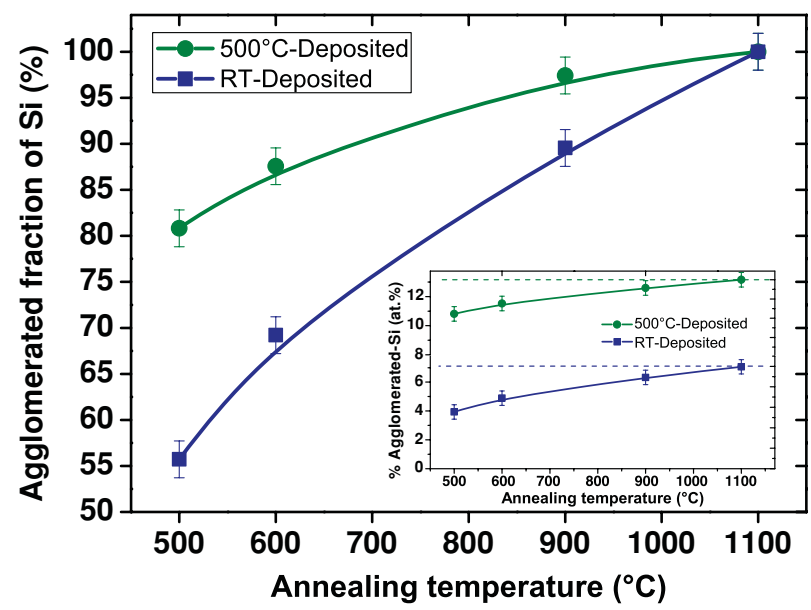

Fig. 4 Compared evolution of the agglomerated fraction of Si normalized to the total Si-excess for RT-deposited (squares) and $500^{\circ} \mathrm{C}$-deposited (circles). Inset: agglomerated-Silicon atoms in atomic percent.

between total $\mathrm{Si}$-excess and agglomerated $\mathrm{Si}$-excess is reduced more and more as $T_{\mathrm{d}}$ is raised, and 3. at $T_{\mathrm{d}}=700^{\circ} \mathrm{C}$, the agglomerated $\mathrm{Si}$ reaches the value of the total excess silicon, indicating a complete phase separation between $\mathrm{Si}$ and $\mathrm{SiO}_{2}$.

This method can also be used to estimate the evolution of the fraction of agglomerated $\mathrm{Si}$ excess as a function of the annealing temperature $T_{\mathrm{a}}$. Two $T_{\mathrm{d}}$ values (RT and $500^{\circ} \mathrm{C}$ ) were chosen to examine the evolution of the fraction of agglomerated $\mathrm{Si}$, as depicted in Fig. 4.

It can be seen in the inset of Fig. 4 that the agglomeration of Si is progressively raising in function of $T_{\mathrm{d}}$ for both kinds of samples, until reaching their highest value after $1100^{\circ} \mathrm{C}$. Indeed, after annealing at $1100^{\circ} \mathrm{C}$, both kinds of samples $\left(T_{\mathrm{d}}\right.$ for RT and $\left.500^{\circ} \mathrm{C}\right)$ reach their limits, 7 at.\% and 13 at.\%, respectively, corresponding to the complete agglomeration of total Si-excess (see inset Fig. 4). The absolute value of $\% \mathrm{Si}_{\text {agglo }}$ in at. $\%$ for RT-deposited is, for each annealing temperature, twice lower than the corresponding one for $500^{\circ} \mathrm{C}$-deposited. This value happens to be governed by the total Si excess content (see Fig. 3).

To compare these two behaviors, we normalize these $\% \mathrm{Si}_{\text {agglo }}$ (at.\%) to their respective total Si-excess ( $7 \%$ for RT; $13 \%$ for $500^{\circ} \mathrm{C}$ ) to obtain the agglomerated fraction (Fig. 4). Both curves show a similar trend toward $T_{\mathrm{a}}$, with a progressive increase of agglomerated $\mathrm{Si}$ and they eventually reach the value of $100 \%$ after $1100^{\circ} \mathrm{C}$-annealing. This does not mean that all excess-Si atoms are located within a Si-nc but any excess $\mathrm{Si}$ atom is bonded to at least another $\mathrm{Si}$ atom. We remark that the fraction of agglomerated $\mathrm{Si}$ is always lower for RT-deposited sample because of its lower as-deposited value of agglomeration compared to the corresponding one for $500^{\circ} \mathrm{C}$-deposited.

\subsection{Cathodoluminescence Spectra}

The CL technique was used for a tentative identification of the optically active centers present within our films. Indeed, since the incident electron beam is able to excite over a wide range of energy, the CL method is able to provide a "mapping" of the different emitting centers. ${ }^{22}$ It is known that even pure $\mathrm{SiO}_{2}$ presents $\mathrm{CL}$ emissions originating from some radiative-defect centers generated by the imperfect local arrangement of atoms, hence deviating from the perfect $\mathrm{SiO}_{4}$ tetraedra. ${ }^{23}$ Therefore, to analyze our SRSO:Er samples, it appears essential to compare their $\mathrm{CL}$ characteristics to those of sputtered $\mathrm{SiO}_{2}, \mathrm{SiO}_{2}$ : $\mathrm{Er}$, and $\mathrm{SRSO}$ samples. The corresponding CL spectra recorded in the visible range are displayed in Fig. 5. 


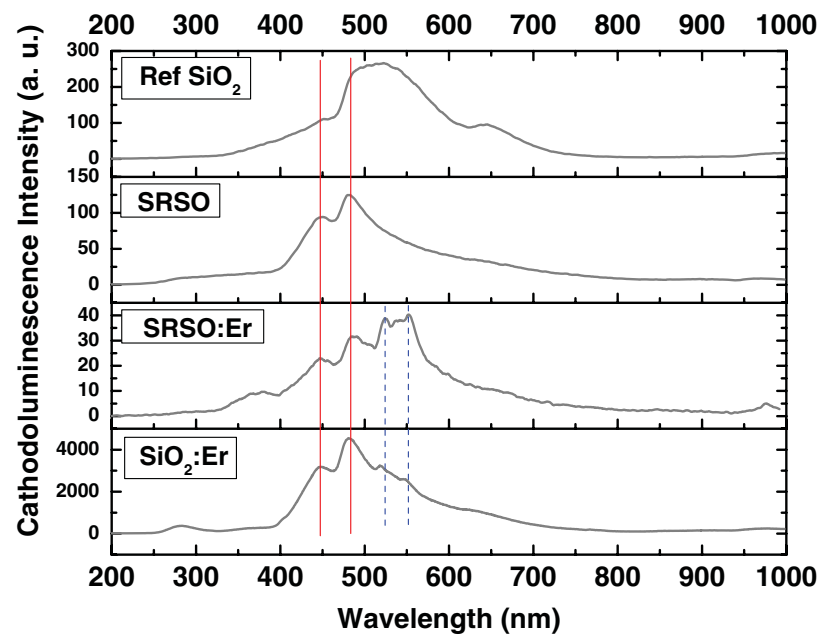

Fig. 5 Cathodoluminescence spectra of (from the top to the bottom): a reference sputtered $\mathrm{SiO}_{2}$, SRSO sample, SRSO:Er sample, and $\mathrm{SiO}_{2}:$ Er. All these samples are nonannealed and were deposited at $500^{\circ} \mathrm{C}$.

We can see that the sputtered $\mathrm{SiO}_{2}$ layer, considered as a reference here, present a broad CL spectra between 300 and $800 \mathrm{~nm}$. This large band is the sum of different contributions, such as oxygen vacancies [in this case oxygen deficient center (ODC)] and nonbridging oxygen hole centers (NBOHC) ${ }^{24}$ This kind of CL spectrum was already observed by other research groups ${ }^{22-25}$ and recently by our group. ${ }^{26}$ When excess silicon is introduced, as for a silicon-rich silicon oxide (SRSO) sample, the corresponding CL spectrum presents two peaks centered at 450 and $480 \mathrm{~nm}$, typically due to ODC. ${ }^{23}$ The Si excess in this material is liable to favor the creation of Silicon-ODC (SiODC), but seems unfavorable for the formation of NBOHC that are, indeed, not detected here. When Er ions are incorporated, as for the SRSO:Er sample, the two SiODC peaks are still emitting at the exact same wavelengths, in addition to two peaks at 520 and $550 \mathrm{~nm}$. As the only difference between this sample and SRSO is the presence of $\mathrm{Er}^{3+}$, these peaks are likely to originate from $\mathrm{Er}^{3+}$ levels' transitions. This hypothesis is further confirmed by the $\mathrm{CL}$ spectra of $\mathrm{SiO}_{2}$ : Er that presents the same transitions that are still distinguishable in spite of the wide overlapping of SiODC contributions. One can, therefore, argue that these two Er-related emissions are respectively arising from the ${ }^{2} \mathrm{H}_{11 / 2}=>{ }^{4} \mathrm{I}_{15 / 2}$ and the ${ }^{4} \mathrm{~S}_{3 / 2}=>{ }^{4} \mathrm{I}_{15 / 2}$ transitions.

Another important feature deserves to be noted: the Er-related emissions are predominant in SRSO:Er sample, at the expense of the SiODC contributions. Indeed, these SiODC emissions in a SRSO:Er are about two orders of magnitude lower than the corresponding ones in a $\mathrm{SiO}_{2}: \mathrm{Er}$ sample free from Si excess. This counterbalancing phenomenon is reminiscent of the earlier mentioned and well known coupling between the $\mathrm{Er}^{3+}$ ions and the Si-based sensitizers. One can therefore consider that we are dealing with a similar energy transfer from SiODC toward $\mathrm{Er}^{3+}$ ions, especially as this is accompanied by a drastic decrease of the emissions from SiODC. These emitting centers appear very similar to the so-called Luminescent Centers of Savchyn et al., ${ }^{16}$ described as oxygen vacancies and which play a predominant sensitizing role toward $\mathrm{Er}^{3+}$ ions. Besides, such an energy transfer was already shown in SRSO from Si-based-entities to neodymium ions, ${ }^{27}$ these Si-based entities have absorption energies very close to those of SiODC. We should also note that a so-called "inverse energy transfer" from $\mathrm{Er}^{3+}$ ions toward Germanium oxygen deficient centers (GeODC) was reported by Kanjilal et al., ${ }^{28}$ indicating that energy transfer between erbium and ODC is possible.

To check the evolution of all emitting centers in function of both $T_{\mathrm{d}}$ and $T_{\mathrm{a}}$, we made specific CL analyses on our samples. Figure 6 reports typical evolution of the CL spectra 

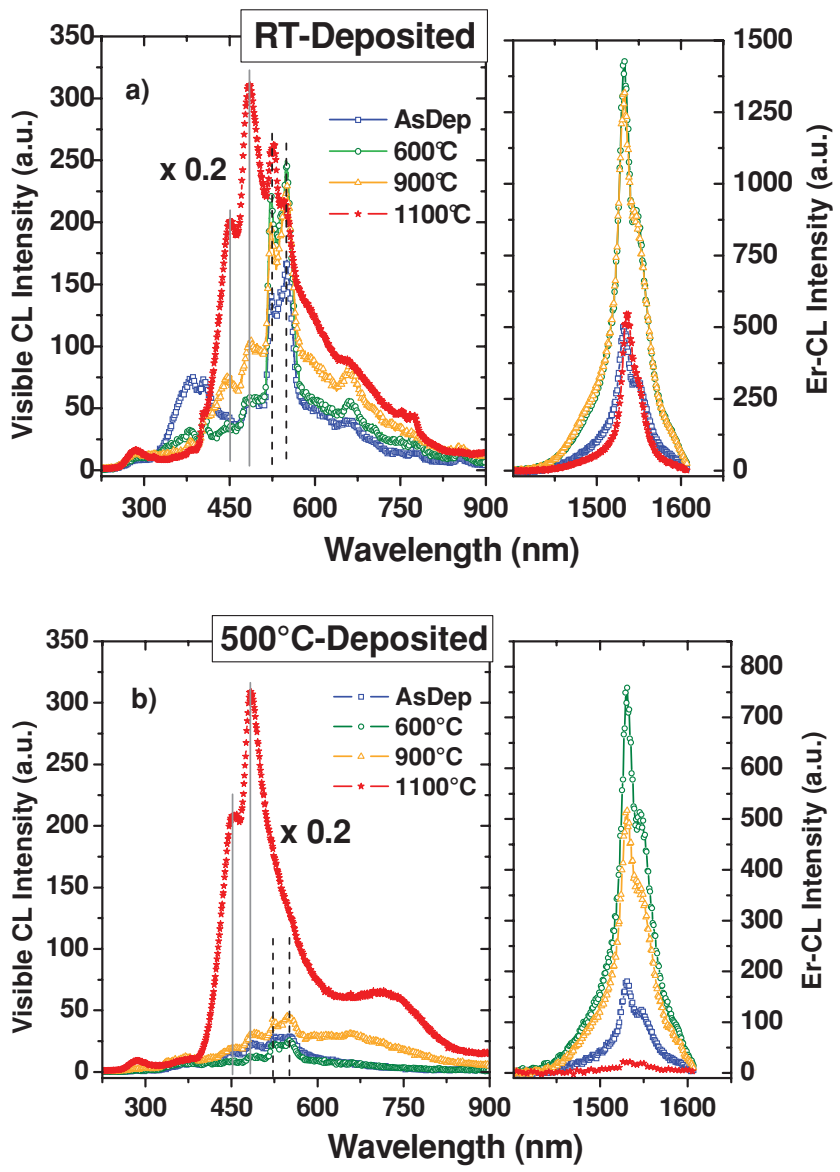

Fig. 6 Cathodoluminescence spectra (normalized to the thickness), according to the temperature of annealing SRSO:Er for samples deposited at (a) room-temperature and (b) $500^{\circ} \mathrm{C}$. The $\mathrm{CL}$ spectra of $1100^{\circ} \mathrm{C}$-annealed samples are divided by five for clarity.

recorded on the as-deposited samples (at $\mathrm{RT}$ and $500^{\circ} \mathrm{C}$ ) and subsequently annealed at 600,900 , and $1100^{\circ} \mathrm{C}$.

First of all, we can see that all samples present the $1.5 \mu \mathrm{m} \mathrm{Er}^{3+}$ emission due to ${ }^{4} \mathrm{I}_{13 / 2}=>{ }^{4} \mathrm{I}_{15 / 2}$ radiative transition, which similarly evolves for both RT-deposited and $500^{\circ} \mathrm{C}$ deposited samples: an intensity increase by a factor of 3-4 until an optimum $T_{\mathrm{a}}=600^{\circ} \mathrm{C}$, followed by a strong decrease after annealing at $1100^{\circ} \mathrm{C}$. Note that the maximum CL intensity (after $600^{\circ} \mathrm{C}$ annealing) for the RT-deposited sample is twice higher than that for $500^{\circ} \mathrm{C}$ deposited one, which can be partly explained by the $20 \%$ less Er in the latter sample (see Fig. 1). But the most significant difference between the two kinds of samples is that the $500^{\circ} \mathrm{C}$-deposited sample contains a total Si-excess twice higher than the RT-deposited one. Thus, the origin of the lowering of $\mathrm{Er}^{3+}$ emission at $1.5 \mu \mathrm{m}$ may be linked to this difference.

By comparing the two CL spectra in the visible range [left panels of Figs. 6(a) and 6(b)], one can observe the following: 1. the Er-related emissions $\left({ }^{2} \mathrm{H}_{11 / 2}=>{ }^{4} \mathrm{I}_{15 / 2}\right.$ and ${ }^{4} \mathrm{~S}_{3 / 2}=>$ ${ }^{4} \mathrm{I}_{15 / 2}$ ) from the RT-deposited samples are $80-90 \%$ higher than those from the $500^{\circ} \mathrm{C}$-deposited ones, 2. the CL contribution assigned to quantum-confinement in Si-nc and observed in $500^{\circ} \mathrm{C}$ deposited samples (at $\sim 650 \mathrm{~nm}$ for $T_{\mathrm{a}}=900^{\circ} \mathrm{C}$ and at $\sim 720 \mathrm{~nm}$ for $T_{\mathrm{a}}=1100^{\circ} \mathrm{C}$ ) is absent for RT-deposited samples, and 3. the visible emissions from $\mathrm{Er}^{3+}$ ions $(520$ and $550 \mathrm{~nm}$ ) are still detected after annealing at $1100^{\circ} \mathrm{C}$ of RT-deposited sample, whereas they are not distinguishable for the $500^{\circ} \mathrm{C}$-deposited one. These observations well confirm that the weak emission of erbium in $500^{\circ} \mathrm{C}$-deposited samples (both in Vis and IR range) is concomitant with a significant emission 
from Si-nc, whereas the RT-deposited samples present high emission from $\mathrm{Er}^{3+}$ but no Si-nc emission. We thus argue that the Si-nc influence the $\mathrm{CL}$ of $\mathrm{Er}^{3+}$ ions, in a way that differs from the PL experiments dealing with the usual energy transfer from Si-nc toward Er that enhances the $\mathrm{Er}^{3+} \mathrm{PL}$. The picture here is more complex as the layers are excited by hot electrons, thus enabling the simultaneous excitation of SiODC, Si-nc, and $\mathrm{Er}^{3+}$ ions. These concurrent impact excitations are likely to provoke simultaneous energy transfer from $\mathrm{Si}-\mathrm{nc}$ toward $\mathrm{Er}^{3+}$ ions as well as energy back-transfer from $\mathrm{Er}^{3+}$ ions toward Si-nc, thus reducing the overall emission from $\mathrm{Er}^{3+}$ ions.

On the other hand, the SiODC contribution becomes predominant in CL spectra for both RT- and $500^{\circ} \mathrm{C}$-deposited samples after a $1100^{\circ} \mathrm{C}$-annealing. In both cases, the huge increase of $\mathrm{SiODC}$ emission is concomitantly occurring with a strong decrease in the $\mathrm{Er}^{3+}$ ions emission at $1.5 \mu \mathrm{m}$. This counterbalancing behavior of the emissions from SiODC and Er ions is indicative of the possible active role of SiODC as sensitizers for $\mathrm{Er}^{3+}$ ions. In addition, the emission from those point-defect centers in the visible range overlaps with the Si-nc absorption band, and this strongly suggests that they may also act as sensitizers for Si-nc. The highest emission of $\mathrm{SiODC}$ (after $1100^{\circ} \mathrm{C}$-annealing) occurs when the $\mathrm{Si}$ excess is completely agglomerated (see Fig. 4), indicating that the SiODC emission is not due to isolated $\mathrm{Si}$ atoms. Besides, at this high annealing temperature providing a complete phase separation between $\mathrm{Si}$ and $\mathrm{SiO}_{2},{ }^{29}$ we assume that SiODC are mainly located at the substoichiometric interface between Si-nc and $\mathrm{SiO}_{2}$ matrix.

These different processes of energy transfer, whether demonstrated or likely to occur in CL experiment, are sketched in Fig. 7 with a proposed scheme of excitation, together with the band diagrams of all considered emitting centers (SiODC, Si-nc, and $\mathrm{Er}^{3+}$ ions).

These CL results give very valuable insights on the presence of all the emitting centers within SRSO:Er layers, as well as the possible processes of energy transfer between the active

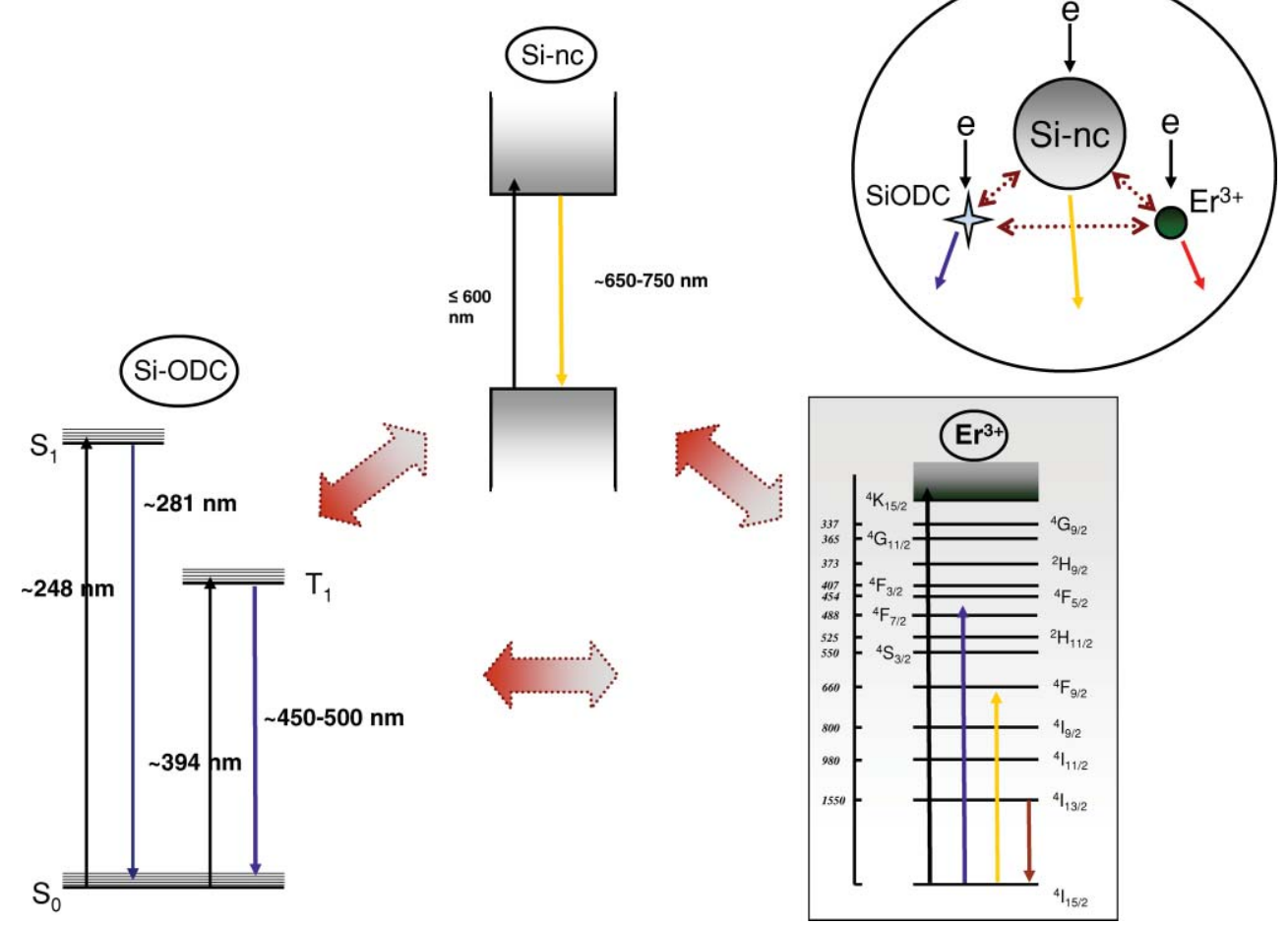

Fig. 7 Schematic band diagrams of all detected emitting centers within SRSO:Er layers. In the upper right part a model of interaction between luminescent entities is depicted. 


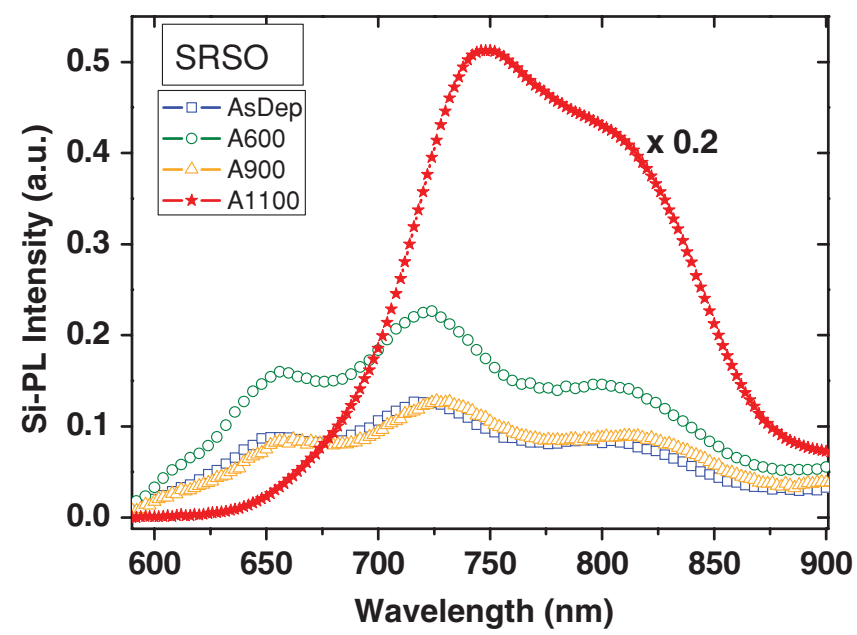

Fig. 8 Photoluminescence results (normalized to film thickness and total Si-excess) on $500^{\circ} \mathrm{C}$ deposited SRSO sample according to the annealing temperature.

emitting centers. To distinguish between these processes, we carried out PL experiments, as complementary to CL studies.

\subsection{Photoluminescence Spectra}

In this part, all PL spectra were obtained using an excitation wavelength $(476 \mathrm{~nm})$ that is nonresonant for $\mathrm{Er}^{3+}$ ions and is well beyond the excitation threshold wavelength for SiODC. This ensures that all emitting $\mathrm{Er}^{3+}$ ions are excited by only Si-nc, ruling out any eventual energy transfer from SiODC to $\mathrm{Er}^{3+}$ ions. In this way, the following interpretations for the energy transfer would be much simpler. Note that an interference pattern is observed for all of the following PL spectra of samples in the range $600-900 \mathrm{~nm}$, because of the reflections occurring at the interfaces of these relatively thick layers. ${ }^{30}$

In order to thoroughly analyze the evolution of the Si-PL emission of SRSO:Er versus $T_{\mathrm{a}}$, a first study was carried out for PL emission of SRSO. Figure 8 shows the evolution of Si-nc-PL emission for SRSO layer free from any $\mathrm{Er}^{3+}$ ions. It can be seen that the influence of the annealing temperature on Si-nc PL emission is not straightforward. The PL intensity is slightly increased from as-deposited to $600^{\circ} \mathrm{C}$ annealing, followed by a decrease after $900^{\circ} \mathrm{C}$ annealing and eventually a fifty-time enhancement after $1100^{\circ} \mathrm{C}$-annealing.

Since no significant shift is observed in the wavelength of the PL spectra for as-deposited and $600^{\circ} \mathrm{C}$-annealed samples, we assume that, although more optically active Si-nc are formed with annealing, their average size remains unchanged. On the contrary the annealing from 600 to $900^{\circ} \mathrm{C}$ leads to a slight shift of the PL spectra, together with a global decrease of the PL intensity. This might be due to some increase of the average size at the expense of their density. This latter phenomenon is strongly increased after $1100^{\circ} \mathrm{C}$ annealing, giving rise to well crystallized Si-nc that were observed by electron microscopy (not shown), responsible of the strong enhancement of Si-nc emission.

Figure 9 displays the PL spectra (normalized to the thickness and to the total Si-excess percentage) in the visible range for both RT- [Fig. 9(a)] and 500 ${ }^{\circ}$-deposited [Fig. 9(b)] samples, subsequently annealed at the indicated temperatures. This visible PL is assumed to originate from the Si-nc not coupled to $\mathrm{Er}^{3+}$ ions, and then not concerned by the energy transfer. Thus, the evolution of the visible band with $T_{\mathrm{a}}$ can be due to a variation in the number of emitting Si-nc and also to an evolution of the coupling between Si-nc and $\mathrm{Er}^{3+}$. For the as-deposited [blue lines in Figs. 8, 9(a), and 9(b)], we can see that the visible PL intensities for RT-deposited 


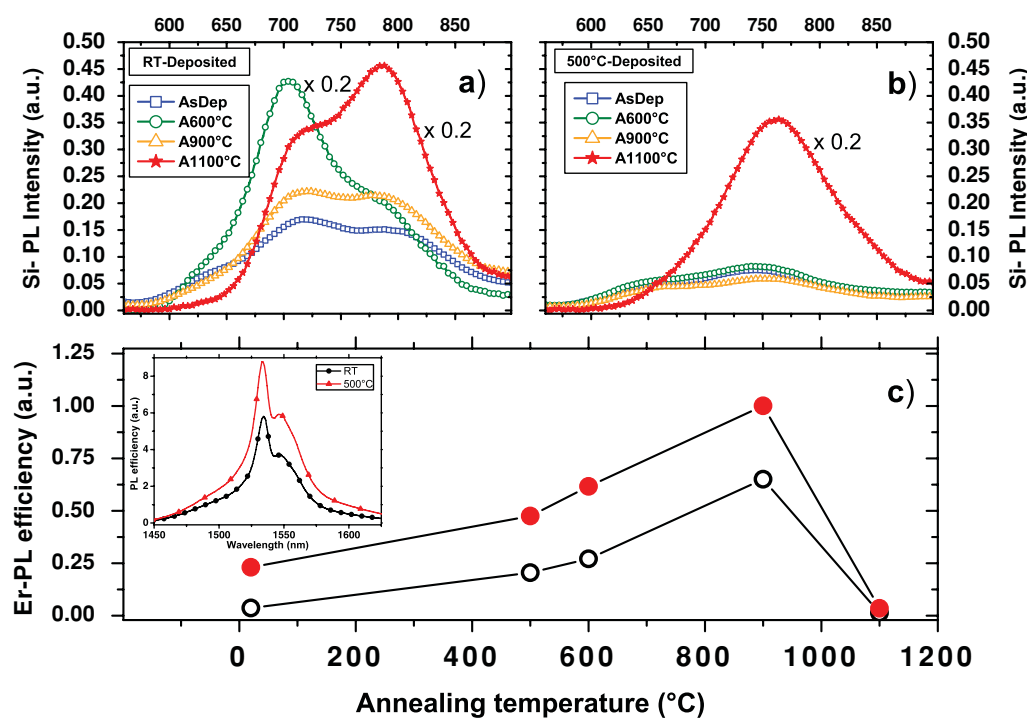

Fig. 9 Photoluminescence results on RT- and $500^{\circ} \mathrm{C}$-deposited samples. Displayed in the upper part are the visible spectra (normalized to the thickness and the atomic percentage of excess-Si) for (a) RT- and (b) $500^{\circ} \mathrm{C}$-deposited samples. (c) Evolution of the Er-PL efficiency at $1.53 \mu \mathrm{m}$ for RT- (empty circles) and $500^{\circ} \mathrm{C}$-deposited (full circles) samples according to the annealing temperature. Inset: Typical spectra of RT- (black) and $500^{\circ} \mathrm{C}$-deposited (red) both annealed at $900^{\circ} \mathrm{C}$.

samples of SRSO:Er and SRSO deposited at $500^{\circ} \mathrm{C}$ are comparable, whereas this intensity is twice lower for the SRSO:Er deposited at $500^{\circ} \mathrm{C}$. This indicates that the energy transfer toward $\mathrm{Er}^{3+}$ ions is more efficient for this latter sample, reducing the overall visible PL band.

The evolution of Si-nc PL against $T_{a}$ for both RT- and $500^{\circ} \mathrm{C}$-deposited SRSO:Er samples, is qualitatively similar to that of SRSO. We thus assume that the evolution of Si-nc PL intensity is mainly governed by the density of Si-nc, and not by their coupling with $\mathrm{Er}^{3+}$ ions. In any case, the Si-nc PL intensity is lower for $500^{\circ} \mathrm{C}$-deposited sample than for the RT-deposited one throughout the annealing temperature range, and this suggests that the energy transfer is always more efficient for $500^{\circ} \mathrm{C}$-deposited sample.

Let us examine the evolution against $T_{d}$ and $T_{a}$ of the corresponding Er-PL that is governed by the excitation through the Si-nc.

Figure 9(c) displays the evolution with $T_{\mathrm{a}}$ of the Er-PL efficiency, defined as the ratio $I_{P L}^{E r} /[E r], I_{P L}^{E r}$ being the Er-PL intensity. It can be seen that the PL efficiency evolves similarly for the two samples deposited at RT and $500^{\circ} \mathrm{C}$. They show both a gradual increase up to a maximum for $T_{\mathrm{d}}=900^{\circ} \mathrm{C}$, before collapsing together when $T_{\mathrm{d}}$ reaches $1100^{\circ} \mathrm{C}$. This evolution with $T_{\mathrm{a}}$ is qualitatively similar to that of CL (Fig. 6), except for the optimum value of $T_{\mathrm{a}}: 900^{\circ} \mathrm{C}$ for PL and $600^{\circ} \mathrm{C}$ for CL. As we are dealing with the same samples for both studies, one can assume that the different optimum $T_{\mathrm{a}}$ may be due to the different excitation sources, electrons for CL and photons for PL. To note, however, that this PL efficiency is systematically higher for the $500^{\circ} \mathrm{C}$-deposited sample, which is quite consistent with earlier observations on samples as-deposited at different $T_{\mathrm{d}}$ values ${ }^{14}$ and with the above-mentioned lower Si-nc emission from $500^{\circ} \mathrm{C}$-deposited samples. While the annealing at the optimum $T_{\mathrm{a}}$ $=900^{\circ} \mathrm{C}$ improves by a factor of four, the PL efficiency for the $500^{\circ} \mathrm{C}$-deposited sample, the corresponding improvement for the RT-deposited layer is improved by a factor of 18 , but still insufficient to reach the $500^{\circ} \mathrm{C}$-deposited Er-PL efficiency. The increase of the PL efficiency is due to an enhanced coupling between the Er ions and the Si-nc sensitizers whose formation is increasingly favored by the annealing up to $900^{\circ} \mathrm{C}$, as claimed above. The earlier reported 


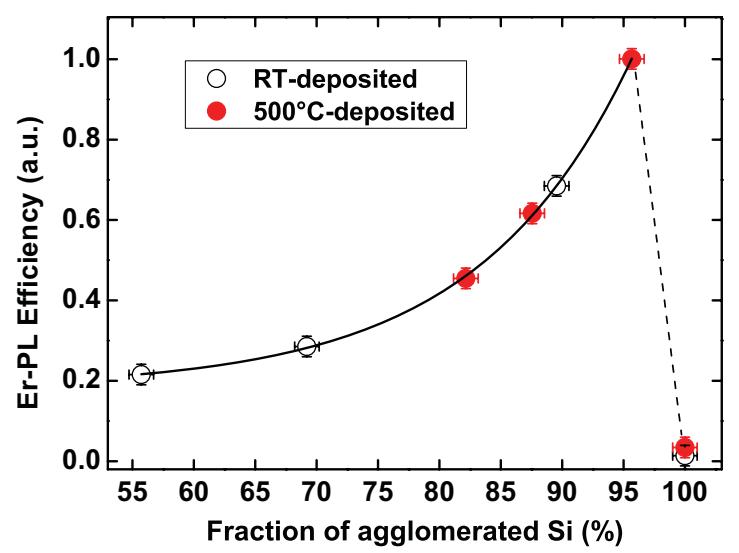

Fig. 10 Evolution of the Er-PL efficiency against the fraction of agglomerated Si. The curve is a guide for the eyes.

increase of the optically active ions with the thermal budget provided an additional support to our explanation. ${ }^{14}$ For the highest value of $T_{\mathrm{d}}\left(1100^{\circ} \mathrm{C}\right)$, two processes compete to the observed drastic decrease of the PL efficiency: first, the tendency of Er to agglomerates, ${ }^{31,32}$ second, the coalescence of Si-nc in large crystallized nanograins, as confirmed by the HRTEM observations of our present samples (not shown). It is worth noting that, although the samples deposited at two different temperatures show generally similar evolutions of Er-PL and Er lifetimes (not shown), the sample grown at $500^{\circ} \mathrm{C}$ shows much better Er emission for each annealing temperature. The origin of this performance is due to the fact that much more $\mathrm{Si}$ atoms are agglomerated during the growth, compared to RT deposited layer. As shown in Figs. 34, the fraction of agglomerated $\mathrm{Si}$ is increasing with $T_{\mathrm{a}}$ and $T_{\mathrm{d}}$. To have a deeper insight on the influence of this fraction on the sensitization of $\mathrm{Er}^{3+}$ ions, in Fig. 10 we display the evolution of the Er-PL efficiency in function of the fraction of agglomerated Si.

We can see that whatever the parameters, such as the total Si-excess and the temperatures of deposition and annealing, the Er-PL efficiency seems to be governed by the fraction of agglomerated Si. Note that the last point shows a collapse of the Er-PL efficiency that is explained by the agglomeration of $\mathrm{Er}^{3+}$ ions, ${ }^{31,32}$ occurring after a $1100^{\circ} \mathrm{C}$-annealing. This result well demonstrates that the fraction of agglomerated $\mathrm{Si}$ is a key factor for the enhancement of the erbium-PL. Future studies will be needed to have a deeper insight on the agglomeration of $\mathrm{Si}$, especially the size and density of Si-nc must be investigated to understand their respective influence on the Er-PL.

\section{Conclusion}

The optical and structural properties of Er-doped silicon rich silicon oxide thin films grown by magnetron cosputtering were investigated in function of both deposition and annealing temperatures. The temperature of deposition was shown to have several crucial effects on the properties of the deposited thin film. When the deposition temperature is raised, an increase of the fraction of agglomerated $\mathrm{Si}$ is observed concomitantly to a lowering of the Er content. Besides, the annealing temperature was shown to increase the fraction of agglomeratedSi until complete phase separation at $1100^{\circ} \mathrm{C}$, regardless of the deposition temperature and the total Silicon-excess content. The cathodoluminescence technique was used to investigate the emission of optically-active centers. It was shown that silicon-oxygen deficient centers emit around 450-500 $\mathrm{nm}$ in all kinds of samples and are likely to contribute to the energy transfer toward both Si-nc and $\mathrm{Er}^{3+}$ ions. To rule out any influence of the SiODC on the coupling of $\mathrm{Er}^{3+}$ ions, the nonresonant $476 \mathrm{~nm}$ wavelength was used for photoluminescence 
experiments. It was shown that the Er-PL efficiency is systematically higher for the $500^{\circ} \mathrm{C}$ deposited samples compared to the RT-deposited ones. It was also observed that the fraction of agglomerated-Si governs the Er-PL efficiency, whatever the value of deposition or annealing temperature.

\section{References}

1. M. Paniccia, "Integrating silicon photonics," Nature Photon. 4, 498-499 (2010).

2. A. J. Kenyon, P. F. Trwoga, M. Federighi, and C. W. Pitt, "Optical properties of PECVD erbium-doped silicon-rich silica: evidence for energy transfer between silicon microclusters and erbium ions," J. Phys.: Condens. Matter 6, L319 (1994).

3. M. Fujii, M. Yoshida, Y. Kanzawa, S. Hayashi, and K. Yamamoto, "1.54 $\mu \mathrm{m}$ photoluminescence of Er doped into SiO films containing Si nanocrystals: Evidence for energy transfer from Si nanocrystals to Er," Appl. Phys. Lett. 71, 1198 (1997).

4. G. Franzò, V. Vinciguerra, and F. Priolo, "The excitation mechanism of rare-earth ions in silicon nanocrystals," Appl. Phys. A: Mater. Sci. Process. 3, 69 (1999).

5. F. Gourbilleau, M. Levalois, C. Dufour, J. Vicens, and R. Rizk, "Optimized conditions for an enhanced coupling rate between Er ions and Si nanoclusters for an improved 1.54- $\mu \mathrm{m}$ emission," J. Appl. Phys. 95, 3717 (2004).

6. J. Lee, J. H. Shin, and N. Park, "Optical gain at $1.5 \mu \mathrm{m}$ in nanocrystal Si-sensitized Er-doped silica waveguide using top-pumping $470 \mathrm{~nm}$ LEDs," J. Lightwave Technol. 23, 19 (2005).

7. H.-S. Han, S.-Y. Seo, and J. H. Shin, "Coefficient determination related to optical gain in erbium-doped silicon-rich silicon oxide waveguide amplifier," Appl. Phys. Lett. 81, 3720 (2002).

8. J. H. Jhe, J. H. Shin, K. J. Kim, and D. W. Moon, "The characteristic carrier-Er interaction distance in Er-doped a-Si/SiO superlattices formed by ion sputtering," Appl. Phys. Lett. 82, 4489 (2003).

9. B. Garrido, C. Garcia, P. Pellegrino, D. Navarro-Urrios, N. Daldosso, L. Pavesi, F. Gourbilleau, and R. Rizk, "Distance dependent interaction as the limiting factor for Si nanocluster to Er energy transfer in silica," Appl. Phys. Lett. 89, 163103 (2006).

10. F. Gourbilleau, C. Dufour, R. Madelon, and R. Rizk, "Effects of Si nanocluster size and carrier-Er interaction distance on the efficiency of energy transfer," J. Lumin. 126, 581 (2007).

11. I. Izeddin, A. S. Moskalenko, I. N. Yassievich, M. Fujii, and T. Gregorkiewicz, "Nanosecond dynamics of the near-infrared photoluminescence of Er-doped $\mathrm{SiO}_{2}$ sensitized with $\mathrm{Si}$ nanocrystals," Phys. Rev. Lett. 97, 207401 (2006).

12. D. Navarro-Urrios, Y. Lebour, O. Jambois, B. Garrido, A. Pitanti, N. Daldosso, L. Pavesi, J. Cardin, K. Hijazi, L. Khomenkova, F. Gourbilleau, and R. Rizk, "Optically active $\mathrm{Er}^{3+}$ ions in $\mathrm{SiO}_{2}$ codoped with Si nanoclusters," J. Appl. Phys. 106, 093107 (2009).

13. K. Hijazi, R. Rizk, J. Cardin, L. Khomenkova, and F. Gourbilleau, "Towards an optimum coupling between Er ions and Si-based sensitizers for integrated active photonics," J. Appl. Phys. 106, 024311 (2009).

14. S. Cueff, C. Labbé, J. Cardin, J.-L. Doualan, L. Khomenkova, K. Hijazi, O. Jambois, B. Garrido, and R. Rizk, "Efficient energy transfer from Si-nanoclusters to Er ions in silica induced by substrate heating during deposition," J. Appl. Phys. 108, 064302 (2010).

15. D. Kuritsyn, A. Kozanecki, H. Przybylinska, and W. Jantsch, "Defect-mediated and resonant optical excitation of $\mathrm{Er}^{3+}$ ions in silicon-rich silicon oxide," Appl. Phys. Lett. 83, 4160 (2003).

16. O. Savchyn, F. R. Ruhge, P. G. Kik, R. M. Todi, K. R. Coffey, H. Nukala, and H. Heinrich, "Luminescence-center-mediated excitation as the dominant Er sensitization mechanism in Er-doped silicon-rich SiO2 films," Phys. Rev. B 76, 195419 (2007). 
17. M. Fujii, M. Yoshida, S. Hayashi, and K. Yamamoto, "Photoluminescence from $\mathrm{SiO}_{2}$ films containing Si nanocrystals and Er: Effects of nanocrystalline size on the photoluminescence efficiency of $\mathrm{Er}^{3+}$," J. Appl. Phys. 84, 4525 (1998).

18. A. Pitanti, D. Navarro-Urrios, N. Prtljaga, N. Daldosso, F. Gourbilleau, R. Rizk, B. Garrido, and L. Pavesi, "Energy transfer mechanism and Auger effect in $\mathrm{Er}^{3+}$ coupled silicon nanoparticle samples," J. Appl. Phys. 108, 053518 (2010).

19. G. Franzò, S. Boninelli, D. Pacifici, F. Priolo, F. Iacona, and C. Bongiorno, "Sensitizing properties of amorphous Si-clusters on the 1.54- $\mu$ m luminescence of Er in Si-rich $\mathrm{SiO}_{2}$," Appl. Phys. Lett. 82, 3871 (2003).

20. T. Sekiguchi, "Development of CL for semiconductor research, Part II: Cathodoluminescence study of semiconductor nanoparticles and nanostructures using low-electron-beam energies," Lect. Notes Phys. 588, 53 (2001).

21. P. G. Pai, S. S. Chao, Y. Takagi, and G. Lukovsky, "Infrared spectroscopic study of $\mathrm{SiO}_{\mathrm{x}}$ films produced by plasma enhanced chemical vapor deposition," J. Vac. Sci. Technol. A 4, 689 (1986).

22. B. Dierre, X. Yuan, and T. Sekiguchi, "Low-energy cathodoluminescence microscopy for the characterization of nanostructures," Sci. Technol. Adv. Mater. 11, 043001 (2010).

23. L. Skuja, "Optically active oxygen-deficiency-related centers in amorphous silicon dioxide," J. Non-Cryst. Solids 239, 16-48 (1998).

24. H. Koyama, "Cathodoluminescence study of $\mathrm{SiO}_{2}$," J. Appl. Phys. 51, 2228-2235 (1980).

25. H.-J. Fitting, "How to make silica luminescent?," J. Lumin. 129, 1488-1492 (2009).

26. S. Cueff, C. Labbé, B. Dierre, F. Fabbri, T. Sekiguchi, X. Portier, and R. Rizk, "Investigation of emitting centers in $\mathrm{SiO}_{2}$ co-doped with silicon nanoclusters and $\mathrm{Er}^{3+}$ ions by cathodoluminescence technique," J. Appl. Phys. 108, 113504 (2010)

27. A. Podhorodecki, J. Misiewicz, F. Gourbilleau, J. Cardin, and C. Dufour, "High energy excitation transfer from silicon nanocrystals to neodymium ions in silicon-rich oxide film," Electrochem. Solid-State Lett. 13(3), K26-K28 (2010).

28. A. Kanjilal, L. Rebohle, M. Voelskow, W. Skorupa, and M. Helm, "Enhanced blue-violet emission by inverse energy transfer to the Ge-related oxygen deficiency centers via $\mathrm{Er}^{3+}$ ions in metal-oxide semiconductor structures," Appl. Phys. Lett. 94, 051903 (2009).

29. G. Franzò, M. Miritello, S. Boninelli, R. Lo Savio, M. G. Grimaldi, F. Priolo, F. Iacona, G. Nicotra, C. Spinella, and S. Coffa, "Microstructural evolution of $\mathrm{SiO}_{x}$ films and its effect on the luminescence of Si nanoclusters," J. Appl. Phys. 104, 094306 (2008).

30. R. Ferre, B. Garrido, P. Pellegrino, M. Peralvarez, C. Garcia, J. A. Moreno, J. Carreras, and J. R. Morante, "Optical-geometrical effects on the photoluminescence spectra of Si nanocrystals embedded in $\mathrm{SiO}_{2}$, , J. Appl. Phys. 98, 084319 (2005).

31. A. Polman, D. C. Jacobson, D. J. Eaglesham, Ft. C. Kistler, and J. M. Poate, "Optical doping of waveguide materials by MeV Er implantation," J. Appl. Phys. 70, 3778-3784 (1991).

32. S. Cueff, C. Labbé, J. Cardin, and R. Rizk, "Impact of the annealing temperature on the optical performances of Er-doped Si-rich silica systems," IOP Conf. Ser.: Mater. Sci. Eng. 6, 012021 (2009).

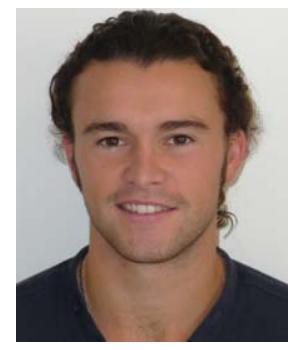

Sébastien Cueff received his engineering degree (equivalent to MSc) in materials and nanotechnology from the National Institute of Applied Sciences (INSA - Rennes), France, in 2008. He is working toward the PhD degree in the CIMAP Laboratory in Caen, France. His current research interests are focused on Silicon-based materials for photonics and optoelectronics applications. Specific work is dedicated to fabrication, characterization (structural, optical, and electrical), and optimization of Erbium-doped Silicon-rich Silicon Oxide thin films. 


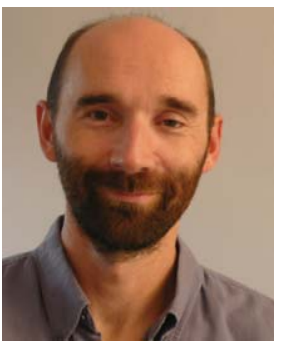

Christophe Labbé is an associate professor in CIMAP (Centre de Recherche sur les Ions, les Matériaux et la Photonique) at Caen, France in the engineering school ENSICAEN. He has obtained his $\mathrm{PhD}$ on rare earth lasers in the Interdisciplinary Research Center Laser Ions at Caen in 1999. He obtained an assistant professor position in the Laboratory of Optical Functions for Telecommunications (FOTON Lab) in 2002 in Rennes. He was specialized in the study of optical and electronic properties of the semiconductors III-V's nano-structures. He based his research on saturable absorbers and became an expert in the self-assembled quantum dot structures in the III-V materials for $1.55 \mu \mathrm{m}$ wavelength laser applications. Since 2008, he joined the CIMAP laboratory to work in spectroscopic characterization group for semiconductor silicon doped rare earth. His on-going research focuses on light sources for telecommunications and on photovoltaic cells. He is currently involved in different research projects for the National Agency of Research in France.

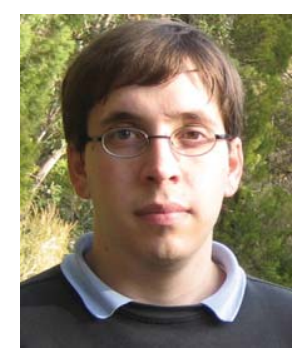

Benjamin Dierre was graduated as an engineer in materials and chemistry from National School of Engineering of Caen (ENSICAEN), France, in 2006. He got a $\mathrm{PhD}$ in materials science and engineering from National Institute for Materials Science (NIMS) and the University of Tsukuba, Japan, in 2010. His research interests include ceramics, semiconductors, electron microscopy, cathodoluminescence, photoluminescence, optoelectronic devices, and electron beam effects on material properties and laser.

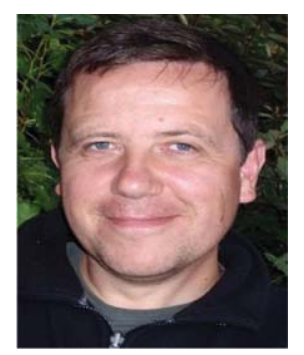

Julien Cardin received the MS and $\mathrm{PhD}$ in solid state physics in 2000 and 2004 from University of Nantes, France. In 2005, he joined the Royal Institute of Technology (KTH) Stockholm for research associate position on development of hybrid photonic devices based on silicon/polymer material. In 2007 he joined the CIMAP laboratory at University of Caen Lower Normandy and contributes to the development of spectroscopic characterization (photoluminescence, electroluminescent, M-lines) of rare earth doped material such as silicon rich silica and Ga2O3. His current research interests are on the development of a ADE-FDTD method devoted to the modeling of electromagnetic interaction with rare earth, Silicon nanograins, and metallic nanoparticles in material developed in CIMAP laboratory.

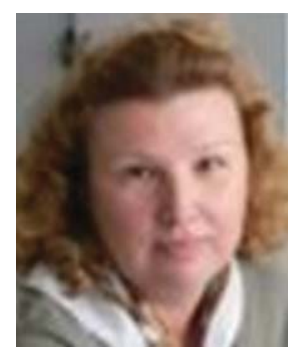

Larysa Khomenkova obtained her MSc degree in technology and materials of microelectronics at Kyiv University (Ukraine) in 1992 and $\mathrm{PhD}$ degree in solid state physics in 1999 from V. Lashkaryov Institute of Semiconductor Physics at National Academy of Sciences of Ukraine (Kyiv, Ukraine). Since 1996 she occupied different research positions in this institute; managed and conducted research projects on II-VI single crystals and nanostructures, as well as on silicon-based nanoscaled materials. In 2007 she joined CIMAP laboratory as an invited researcher, where she carried out the investigation of Si-based nanostructures for photonic and microelectronic applications. Her current research interests include the elaboration of semiconductor and dielectric materials and structures on their basis, optical and luminescent spectroscopy of nanostructures, nonequilibrium processes in solid state materials, and instability and degradation phenomena in the devices. She is the author and coauthor of more than 80 technical papers. 


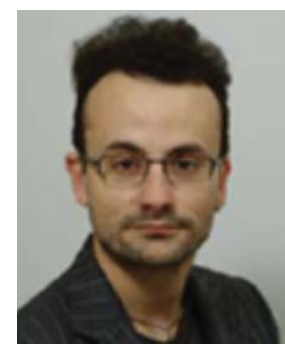

Filippo Fabbri has obtained a PhD from the University of Bologna, Italy, in 2008 studying the effects of neutron irradiation on silicon carbide particle detectors. During the PhD he started a strong collaboration with National Institute for Materials Science (NIMS), Japan. His main topics are the characterization of the optical and electrical properties of nanostructures and nanostructured materials in particular fourth group (mainly silicon and silicon carbide) and metal oxide semiconductors (zinc oxide and titanium dioxide).

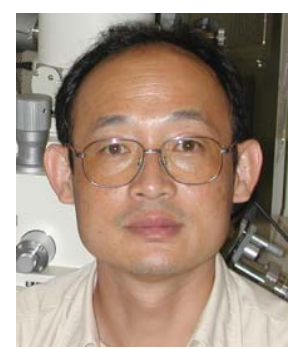

Takashi Sekiguchi has been engaged in the study of defects in semiconductors for 25 years and has published more than 200 papers. He received his $\mathrm{PhD}$ from Tohoku University for the characterization of defects in semiconductors by using electron beam. His main contribution is the cathodoluminescence study of dislocation related luminescence in $\mathrm{Si}$. He has been studying a wide variety of materials from multicrystalline $\mathrm{Si}$ for solar cells to $\mathrm{ZnO}$ nanoparticles.

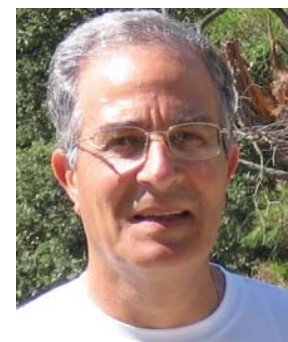

Richard Rizk is deputy director of CIMAP Lab and received the Doctorat d'Etat (1976) in physics at the University of Paris (Orsay Campus). He focused his research on solid-state physics covering the fields of electronics, optoetoelectronic, and photonic Si-based materials and devices. He is currently director of research at the CNRS and has authored or coauthored more than 160 peer-reviewed publications and about 40 invited papers, in addition to tens of contributions to books and special issues. He was coordinator of the EU-funded STREP FP6-LANCER and workpackNetwork NANOMAT). age leader in other EU-funded projects (STREP FP5-SINERGIA and FP4 